\title{
Long-Range Electron Tunneling in Aqueous and Organic Glasses
}

\author{
Oliver S. Wenger§, Harry B. Gray*, and Jay R. Winkler \\ $\S$ Mettler Toledo Award Winner (Oral Presentation)
}

\begin{abstract}
Photoinduced electron transfer (ET) reactions have been investigated in glassy media at $77 \mathrm{~K}$. Distance decay parameters for electron tunneling through water, 2-methyltetrahydrofuran, and toluene have been determined through measurements of donor luminescence quenching by randomly dispersed electron acceptors. Remarkably different long-range ET efficiencies in the three solvents are in accord with the predictions of a superexchange model of distant electronic couplings. We conclude that tunneling energy effects play an important role in long-range ET reactions, and further that the coupling drops off very rapidly across van der Waals gaps between molecules in glasses.
\end{abstract}

Keywords: Charge separation · Electron transfer · Electronic coupling · Superexchange $\cdot$ Tunneling

In photosynthesis, controlled electron transfer (ET) produces distant charge separation that facilitates the storage of solar energy in chemical form [1]. Over the past few decades, investigations of photoinduced ET reactions in a wide range of donor-acceptor systems have shed light on the factors that control the rates of these types of charge separation processes, notably including experimental evidence for the inverted driving force effect in synthetic donor-acceptor molecules [2][3], the design and construction of mimics of the photosynthetic reaction center [4][5], as well as the demonstration that electron tunneling through proteins can occur over distances greater than $15 \AA$ [6]. It also has been shown that charge can travel over distances of $\sim 40 \AA$ in artificial molecular wires [7].

${ }^{*}$ Correspondence: Prof. H.B. Gray, Dr. J.R. Winkler California Institute of Technology

Beckman Institute, M/C 139-74

1200 East California Boulevard

Pasadena, CA 91125, United States

Tel.: +1 6263956500

Fax: + 16264494159

E-Mail: hbgray@caltech.edu; winklerj@caltech.edu www.bilrc.caltech.edu
In very recent work, we have investigated electron tunneling through molecules in aqueous and organic glass matrices [8][9]. The efficiency of solvent as a medium for ET is a relatively unexplored aspect of long-range electron tunneling studies. We would like to know to what extent 'intramolecular' ET is mediated by the solvent that surrounds a given donor-bridge-acceptor system. Early investigations of ET through glassy media [10-12] as well as more recent studies of C-clamp donor-acceptor systems [13] have established that covalent bonds are not required for longrange electron tunneling, and very recently we have studied the distance dependence of photoinduced electron tunneling through water/sulfuric acid, 2-methyltetrahydrofuran (MTHF), and toluene glasses at $77 \mathrm{~K}$ by monitoring donor luminescence quenching by randomly dispersed electron acceptors (Fig. 1a) [8][9]. Since electron donors and acceptors are randomly distributed in the glass matrix (Fig. 1b), there is a statistical distribution of ET rates, leading to highly nonexponential donor luminescence decays from which information on the distance dependence of tunneling through the various glassy media can be extracted. The key requirement for a successful experiment is that the ET reactions must be driving force optimized if they are to be observable in a rigid matrix at $77 \mathrm{~K}$. After much trial and error, we found that $\mathrm{Ru}$ (terpyridine $)_{2}{ }^{2+} /$ $\mathrm{Fe}\left(\mathrm{OH}_{2}\right)_{6}{ }^{3+}$ and $[\operatorname{Ir}(\mu$-pyrazolyl $)(1,5-\mathrm{cy}-$ clooctadiene)],2,6-dichloro-1,4-benzoquinone are suitable donor-acceptor pairs for aqueous and MTHF/toluene glasses, respectively [8][9]. Analysis of the donor luminescence decay data (Fig. 2) for various electron acceptor concentrations [11][14][15] yielded ET distance decay parameters $(\beta)$ for the various glasses (Table). In glassy toluene, $\beta$ is $1.23 \AA^{-1}$; in MTHF and aqueous glasses, the decay constant is $\sim 1.6 \AA^{-1}$. Our experiments show that at a donor-acceptor distance of $20 \AA$, electron tunneling through toluene proceeds $\sim 1000$ times faster than through MTHF or water. What causes this large difference in longrange ET rates?

An acceptable answer to the above question can be obtained from a superexchange model of the electronic coupling between a donor and acceptor separated by a bridge of repeating units [16]. The model predicts a simple relationship between the distance decay parameter $\beta$, the length $\alpha$ of the bridge unit, the electronic coupling between individual bridge elements $h_{b b}$, and the tunneling energy gap $\Delta \varepsilon$ :

$$
\beta=\frac{2}{\alpha} \cdot \ln \left(\frac{\Delta \varepsilon}{h_{b b}}\right)
$$

$\Delta \varepsilon$ is the difference between the energy of the donor-acceptor states at the transition state configuration and the energy of the bridge states. In the aqueous glass, hydrogen bonds will provide relatively strong coupling (large $\mathrm{h}_{\mathrm{bb}}$ ) between individual solvent (bridge) molecules. Nevertheless, the MTHF glass mediates ET equally well, likely due to the combined effect of larger 
(a)

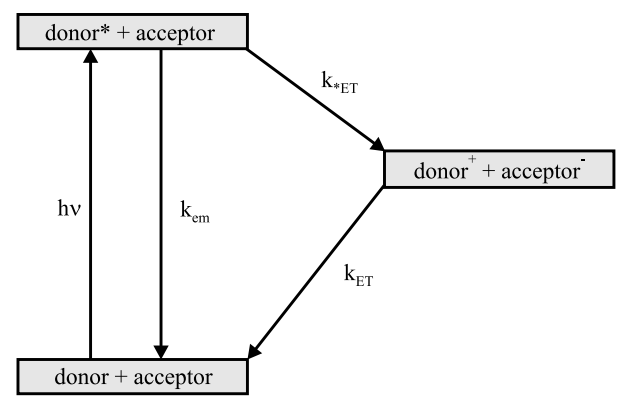

(b)

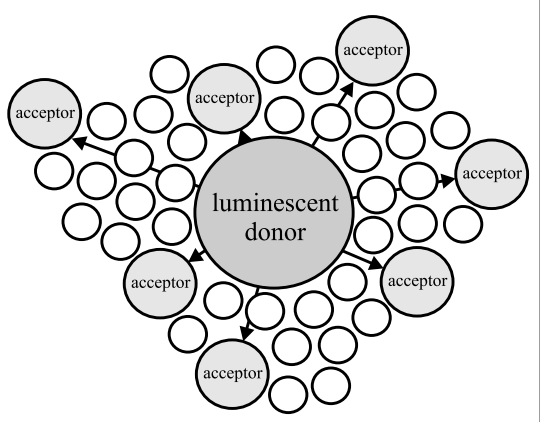

Fig. 1. (a) In the photoinduced ET experiments, donors (Ru(terpyridine) $)_{2}^{2+}$ in $\mathrm{H}_{2} \mathrm{O}_{2} \mathrm{H}_{2} \mathrm{SO}_{4}$ glasses [8][9]; [Ir( $\mu$-pyrazolyl)(1,5-cyclooctadiene) $]_{2}$ in MTHF and toluene glasses [9] are selectively excited (8 ns laser pulse) to long lived $(>\mu \mathrm{s})$ luminescent excited states. Radiative and nonradiative excitedstate depopulation is in competition with ET from these powerful reductants to electron acceptors $\left(\mathrm{Fe}\left(\mathrm{OH}_{2}\right)_{6}{ }^{3+}\right.$ in aqueous; 2,6-dichloro-1,4-benzoquinone in organic glasses). By monitoring the donor luminescence decays $\left(\mathrm{k}_{\mathrm{em}}\right)$, it is possible to obtain excited-state ET rates $\left(\mathrm{k}_{\mathrm{x}_{\mathrm{ET}} \mathrm{T}}\right)$. (b) Donors $(\sim 30 \mu \mathrm{M})$ and acceptors $(0.05-0.5 \mathrm{M})$ are randomly dispersed in glassy solvents. The long donor excited-state lifetime allows photoinduced ET to be examined over distances up to $22 \AA$ [9].
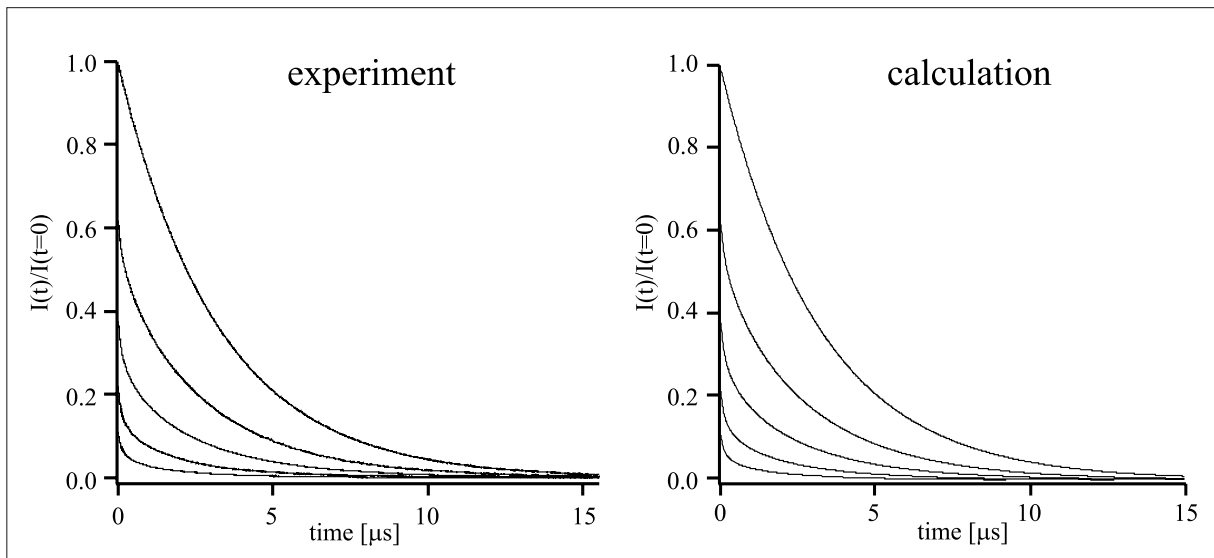

Fig. 2. A wide distribution of donor-acceptor distances produces highly nonexponential luminescence decay curves. Left: experimental $\left[\operatorname{lr}(\mu \text {-pyrazolyl)(1,5-cyclooctadiene) }]_{2}\right.$ luminescence decay kinetics in a MTHF glass at $77 \mathrm{~K}$. The top curve is the single exponential decay obtained in the absence of electron acceptors. With increasing 2,6-dichloro-1,4-benzoquinone concentration $(0.05,0.10,0.15$, $0.20 \mathrm{M}$ ), the decays become increasingly nonexponential. The relative intensities of the decay curves were determined by separate time-integrated luminescence quantum yield measurements. Right: simulated decays using a two-parameter model [14][15]. From fits to the experimental decay data, we obtained a distance decay parameter $\beta=1.62 \AA^{-1}$ for the MTHF glass [9].

Table. Distance decay parameters for electron tunneling through various media

$\begin{array}{ll} & \beta\left[\AA^{-1}\right] \\ \mathrm{H}_{2} \mathrm{O} / \mathrm{H}_{2} \mathrm{SO}_{4} \text { glass } & 1.59 \\ \text { MTHF glass } & 1.62 \\ \text { toluene glass } & 1.23 \\ \text { covalent alkane bridges } & \sim 1.0^{\mathrm{a}} \\ \text { protein } \beta \text {-strand } & 1.1^{\mathrm{b}} \\ \text { oligo-phenylene bridges } & 0.4-0.8^{\mathrm{c}} \\ \text { afrom ref. [20]; bfrom ref. [17]; cfrom refs. } \\ \text { [22][23] }\end{array}$

bridge unit size and smaller tunneling energy gap. The substantially more efficient ET through toluene is attributed to the presence of $\pi$-bonding and antibonding orbitals that lead to a smaller tunneling energy gap than in MTHF or water. In addition, the excess electron or hole will be delocalized over the set of $\pi$ molecular orbitals of the toluene bridge molecules, but in 2-methyltetrahydrofuran and water it will be localized on two or three atoms.

Our work has shown that water is a poor medium for ET: its distance decay parameter of $1.59 \AA^{-1}$ compares to a $\beta$-value of $\sim 1.1 \AA^{-1}$ for tunneling through a polypeptide backbone [16]. Thus, at a donor-accep- tor distance of $20 \AA$, ET through water is prohibitively slow, whereas tunneling over such long distances in proteins occurs with rates on the order of $10^{4} \mathrm{~s}^{-1}$ [18]. In addition, the high dielectric constant of water imposes large reorganization barriers to electron transfer [19]; therefore, exclusion of water from active sites often triggers electron transfers through proteins.

The finding that tunneling through $\operatorname{MTHF}\left(1.62 \AA^{-1}\right)$ is much less efficient than through alkane bridges $\left(\sim 1.0 \AA^{-1}\right)$ [20][21] confirms that electronic coupling across van der Waals gaps is much weaker than through covalent bonds: in a recent report, we estimated that ET through a $2-\AA$ van der Waals gap is $\sim 50$ times slower than tunneling the same distance through a covalently bonded bridge [9]. This finding has important implications for biological electron transfer, as the medium between redox centers in proteins is a heterogeneous array of covalent, hydrogen bonded, and van der Waals contacts between atoms of the polypeptide matrix. The protein secondary and tertiary structure ensures that there exists a tunneling pathway for electrons that involves many covalent and hydrogen bonds such that electron tunneling can occur efficiently over long distances [6].

Comparison of the distance decay parameters for toluene $\left(1.23 \AA^{-1}\right)$ and oligophenylene bridges $\left(0.4-0.8 \AA^{-1}\right)$ [22][23] leads to the same conclusion: there is a significant coupling penalty associated with van der Waals gaps. The finding that toluene is a significantly better ET mediator than MTHF is especially noteworthy, as this is direct experimental confirmation of the superexchange coupling model developed more than forty years ago [16]. Our work suggests that the tunneling energy gap $\Delta \varepsilon$ plays a decisive role in determining long-range electronic couplings; aromatic bridges have lower lying energy levels than saturated organic bridges, and this leads to enhanced electronic coupling between donor and acceptor. The dependence of donor-acceptor coupling on the tunneling energy gap demonstrated in our work could potentially be exploited in synthetic systems if $\Delta \varepsilon$ could be minimized for photoinduced charge separation and at the same time maximized to disfavor thermal charge recombination. A design of this type would represent a key step in the development of efficient artificial photochemical energy storage systems.

\section{Acknowledgments}

This research was supported by BP, NSF (US:CHE-0078809), and the Arnold and Mabel Beckman Foundation. O.S.W. acknowledges a postdoctoral fellowship from the Swiss NSF.

Received: December 23, 2004 
[1] G. Feher, J.P. Allen, M.Y. Okamura, D.C. Rees, Nature 1989, 339, 111.

[2] G.L. Closs, J.R. Miller, Science 1988, 244, 440.

[3] L.S. Fox, M. Kozik, J.R. Winkler, H.B. Gray, Science 1990, 247, 1069.

[4] G. Steinberg-Yfrach, J.-L. Rigaud, E.N. Durantini, A.L. Moore, D. Gust, T.A. Moore, Nature 1998, 392, 479.

[5] A.D. Joran, B.A. Leland, P.M. Felker, A.H. Zewail, J.J. Hopfield, P.B. Dervan, Nature 1987, 327, 508.

[6] H.B. Gray, J.R. Winkler, Q. Rev. Biophys. 2003, 36, 341

[7] W.B. Davis, W.A. Svec, M.A. Ratner, M.R. Wasielewski, Nature 1998, 396, 60.

[8] A. Ponce, H.B. Gray, J.R. Winkler, J. Am. Chem. Soc. 2000, 122, 8187.

[9] O.S. Wenger, B.S. Leigh, R. Villahermosa, H.B. Gray, J.R. Winkler, Science, 2005, 307, 99-102.

[10] J.R. Miller, Science 1975, 189, 221.

[11] J.R. Miller, J.V. Beitz, R.K. Huddleston, J. Am. Chem. Soc. 1984, 106, 5057.
[12] T. Guarr, M. McGuire, S. Strauch, G. McLendon, J. Am. Chem. Soc. 1983, 105, 616.

[13] K. Kumar, Z. Lin, D.H. Waldeck, M.B. Zimmt, J. Am. Chem. Soc. 1996, 118, 243.

[14] M. Inokuti, F. Hirayama, J. Chem. Phys. 1965, 43, 1978.

[15] A. Blumen, J. Chem. Phys. 1980, 72, 2632.

[16] H.M. McConnell, J. Chem. Phys. 1961, $35,508$.

[17] R. Langen, I.J. Chang, J.P. Germanas, J.H. Richards, J.R. Winkler, H.B. Gray, Science 1995, 268, 1733.

[18] J.R. Winkler, B.G. Malmström, H.B. Gray, Biophys. Chem. 1995, 54, 199.

[19] R.A. Marcus, N. Sutin, Biochim. Biophys. Acta 1985, 811, 265.

[20] J.F. Smalley, H.O. Finklea, C.E.D. Chidsey, M.R. Linford, S.E. Creager, J.P. Ferraris, K. Chalfant, T. Zawodzinsk, S.W. Feldberg, M.D. Newton, J. Am. Chem. Soc. 2003, 125, 2004.
[21] H. Oevering, M.N. Paddon-Row, M. Heppener, A.M. Oliver, E. Cotsaris, J.W. Verhoeven, N.S. Hush, J. Am. Chem. Soc. 1987, 109, 3258.

[22] A. Helms, D. Heiler, G. McLendon, J. Am. Chem. Soc. 1992, 114, 6227.

[23] R. Villahermosa, Ph. D. thesis, California Institute of Technology 2002. 